\title{
ORIGINAL
}

\section{IMPACTO SOCIAL Y ECONÓMICO DE LA VACUNACIÓN FRENTE A LA VARICELA A LOS 15 MESES DE EDAD EN CASTILLA Y LEÓN EN 2004}

\author{
A Pérez-Rubio (1), JJ Castrodeza Sanz (1), M Gil Costa (2), FJ Luquero Alcalde (1), J Eiros Bouza (3) \\ y R Ortiz de Lejarazu (3)
}

(1) Servicio de Medicina Preventiva y Salud Pública. Hospital Clínico Universitario. Valladolid.

(2) Observatorio de Salud Pública. Dirección General de Salud Pública y Consumo de Castilla y León.

(3) Servicio de Microbiología. Hospital Clínico Universitario. Valladolid.

\section{RESUMEN}

Fundamento: La varicela es una enfermedad infecciosa fundamentalmente infantil producida por el virus Herpes Varicela Zoster que produce importantes costes sanitarios y sociales. En 2005 Castilla y León introdujo en su calendario de vacunación infantil la vacuna de la varicela a los niños de once años susceptibles de padecerla. Dicha estrategia no modifica la importante morbilidad que genera en edades inferiores. El objetivo de este trabajo es valorar la rentabilidad de la vacunación sistemática frente a la varicela a los niños de 15 meses de edad en Castilla y León.

Métodos: Se ha planteado una evaluación económica de coste-beneficio a través de un árbol de decisión. Se estudia una cohorte ficticia de 100.000 niños castellano-leoneses que en el año 2004 cumplieran 15 meses, a los que se les administraría junto a la vacuna triple vírica la de la varicela. El estudio se plantea desde la perspectiva social. El horizonte temporal elegido ha sido hasta que la cohorte de estudio cumpliera 15 años, aplicando una tasa de descuento del 3\%. Para valorar la incertidumbre de algunas variables se ha desarrollado un análisis de sensibilidad.

Resultados: El coste-beneficio de la introducción de la vacuna en el calendario de vacunación infantil se cifra en 1,23.

Conclusiones: Desde la perspectiva social la estrategia de vacunación frente a la varicela, junto a la triple vírica se muestra rentable. La rentabilidad se ve modificada tanto si se introduce una segunda dosis de vacuna como si se analizan sólo los costes directos sanitarios.

Palabras clave: Análisis coste beneficio. Varicela. Vacunación.

Correspondencia:

Alberto Pérez Rubio

Servicio de Medicina Preventiva y Salud Pública

Hospital Clínico Universitario

C/ Ramón Cajal 3 s/n

47003 Valladolid

Correo electrónico:albertoprz@gmail.com
ABSTRACT

\section{Social and Economic Impact of Chicken Pox Vaccine at 15 Months of Age. Castile and Leon, Spain, 2004}

Background: Chicken pox is a mainly childhood contagious disease caused by the Varicella Zoster Virus which gives rise to major healthcare and social costs. In 2005, Castile and Leon added chicken pox vaccine injections to its childhood vaccination schedule for eleven year-olds subject to coming down with this disease. This strategy does not modify the major mobility generated thereby at younger ages. This study is aimed at evaluating the profitability of systematic vaccination for chicken pox in infants 15 months of age in Castile and Leon.

Methods: An economic cost-benefit evaluation has been set out by jeans of a decision-making tree. A fictitious cohort of 100,000 children in Castile and Leon having reached 15 months of age in 2004 is studied, to whom the chicken pox vaccine would be administered in conjunction with the mumps, measles, rubella vaccines. This study is approached from the social standpoint. The time horizon selected was that of up until the study cohort was to reach 15 years of age, applying a $3 \%$ discount rate. A sensitivity analysis was made for evaluating the uncertainty of some variables..

Results: The cost-benefit ratio of adding this vaccine to the childhood vaccination schedule amounts to 1.23.

Conclusions: From the social standpoint, administering chicken pox vaccine in conjunction with the mumps, measles, rubella vaccines show itself to be profitable. The profitability is modified both if a second dose of vaccine is added as well as if only the direct healthcare costs are analyzed.

Key words: Cost Benefit Analysis. Varicella. Vaccination. 


\section{INTRODUCCIÓN}

La varicela es una enfermedad infecciosa fundamentalmente infantil producida por el Herpes virus Varicela Zoster (VVZ). Está probado que antes de la edad de 5 años, el $50 \%$ de la población ha padecido la enfermedad y a los 15 años, el $90 \%$ ya presenta anticuerpos ${ }^{1}$. Aunque se clasifica como enfermedad benigna, existen complicaciones que se desarrollan no sólo en niños inmunodeprimidos, sino también en inmuno- competentes, tales como sobreinfecciones bacterianas, complicaciones neurológicas, respiratorias, gastrointestinales y osteoarticulares, entre otras ${ }^{2}$.

Diferentes estudios han probado que la varicela genera importantes costes sanitarios y sociales ${ }^{3-4}$. Los niños sanos menores de 12 años de edad suponen cerca del 85\% de las consultas médicas relacionadas con esta enfermedad, el $80 \%$ de las hospitalizaciones, prácticamente el $50 \%$ de los casos mortales y la mayor parte de los costes anuales ${ }^{5}$. Estas investigaciones demográficas, que demostraron el grado de morbilidad de la varicela, sirvieron de justificación, junto con el desarrollo de vacunas inmunógenas, efectivas y seguras ${ }^{6-7}$, para la adopción de una política de vacunación universal.

El conocimiento de la carga que supone la enfermedad, en cada región en particular, es de especial importancia para las autoridades sanitarias a la hora de tomar decisiones acerca de la implantación de programas de Salud Pública y de asignación de recursos sanitarios ${ }^{8}$. En España se han publicado distintos trabajos que evidencian la relevancia social y económica que origina la varicela en las distintas Comunidades de nuestro país, y que inician el debate sobre la estrategia vacunal más adecuada ${ }^{9-12}$.

Distintas comunidades autónomas españolas han introducido la vacunación de la varicela en su calendario infantil. Castilla y
León la introdujo, en el año 2005, a los niños de once años susceptibles. Dicha estrategia trata de evitar únicamente la enfermedad en edades donde las complicaciones son más frecuentes e importantes ${ }^{13}$, sin embargo no se persigue alterar la circulación del virus salvaje ni modificar la carga económica y social que supone la enfermedad, hechos que se podrían conseguir adelantando la edad de vacunación a los 15 meses. El objetivo de este trabajo es valorar la rentabilidad de la vacunación sistemática frente a la varicela a los niños de 15 meses de edad en Castilla y León.

\section{MATERIAL Y MÉTODOS}

Se ha planteado una evaluación económica de coste-beneficio, a través de la representación gráfica de cada una de las estrategias a valorar: vacunación o no vacunación. Las distintas opciones incorporan las probabilidades de las categorías clínicas, y el resultado será el producto de las probabilidades de cada una de ellas.

Se estudia una cohorte ficticia de 100.000 niños castellano-leoneses que en el año 2004 cumplieran 15 meses, y a los que se les administraría junto a la vacuna de la Triple Vírica (TV) la vacuna de la Varicela, frente a los que únicamente recibiría la vacunación TV como plantea el calendario vacunal de Castilla y León. La administración conjunta de ambas vacunas no altera la inmunogenicidad, efectividad y seguridad de las mismas ${ }^{14}$.

El estudio se plantea desde la perspectiva social, en donde se incluyen tanto lo costes tangibles directos sanitarios, afrontados por el proveedor de los servicios sanitarios, SACYL(Servicio Regional de Salud de Castilla y León), como los costes indirectos que ocasiona la enfermedad, que se relacionan sobre todo con pérdidas de productividad de los cuidadores. Los beneficios del programa de vacunación son los ahorros en 
los costes de la enfermedad que se producen como consecuencia de la puesta en marcha del programa, es decir, la disminución de los costes de la enfermedad (costes negativos) como consecuencia de la disminución de la incidencia de la varicela atribuible a la vacunación. El horizonte temporal fijado ha sido hasta que la cohorte de estudio cumpliera 15 años.

Se eligió el año 2004 por la disponibilidad de datos descriptivos de incidencia y características epidemiológicas de la enfermedad, así como de recursos consumidos, recogidos por la Red de Médicos Centinelas de Castilla y León (RMCCyL), que han completado de forma importante el conocimiento de la enfermedad ofrecido por el Sistema de Declaración Obligatoria, en el que la varicela es de declaración exclusivamente numérica ${ }^{15}$.

Los costes directos contemplados en el estudio, debido a la ausencia de publicación de precios públicos a aplicar por los centros sanitarios en la Comunidad de Castilla y León, se obtuvieron mediante consulta directa de diferentes fuentes: Direcciones Técnicas de Atención Primaria y de Atención Especializada de la Gerencia Regional de Salud de la Comunidad de Castilla y León, unidades de contabilidad analítica de hospitales de la provincia de Valladolid y búsqueda bibliográfica de autores españoles. Para el cálculo de los costes indirectos se ha utilizado la media diaria del sueldo medio de la población castellano-leonesa, estimado por el Instituto Nacional de Estadística a través de la encuesta de estructura salarial.

La vacunación de niños menores de 13 años se realiza a través de la vacuna comercializada en España VARIVAX ${ }^{\circledR}$ (Aventis Pasteur MSD). El coste de inmunización incluye, además de la única dosis vacunal, el coste de personal y material necesario para administrarla. Los costes indirectos debidos a la pérdida productiva de algún familiar por acompañar a los niños durante las visitas de vacunación no se contemplan, debido a que ésta se administra junto a la vacuna triple vírica. El coste de la vacuna, obtenido del precio aplicado a la Comunidad de Castilla y León para el desarrollo de la actual campaña de vacunación, es de 32 euros, y el coste de su administración es de 2,5 euros, con un total de 34,5 euros por persona.

\section{Cobertura y efectividad de la vacunación (tabla 1)}

La vacuna se compone de una única dosis de $0,5 \mathrm{ml}$, con una eficacia vacunal del $100 \%$ durante el primer año ${ }^{16}$. La protección frente a la enfermedad (efectividad vacunal) varía entre el $71 \%$ al $100 \%$, con unas estimaciones medias en torno al $86 \%$ para todo tipo de varicela y entre $90 \%$ $100 \%$, para la varicela moderada o grave $^{17,18}$. Estos hallazgos son consistentes con las observaciones de otros estudios en los que el riesgo de "breakthrough" (fallo vacunal) aumenta con el tiempo y con una edad menor de 15 meses en el momento de la vacunación ${ }^{19,20}$. El seguimiento de los efectos secundarios de la vacuna, realizado durante un período en el que se administraron casi 10 millones de dosis, ha demostrado la seguridad de la misma ${ }^{7}$. La duración de la protección proporcionada por la vacuna durante el período de estudio elegido ha sido documentada en distintos estudios ${ }^{21,22}$. Se ha asumido una cobertura vacunal del $100 \%$, ya que el porcentaje de vacunados con la primera dosis de TV (triple vírica) en Castilla y León es del 97,83\%, existiendo provincias que alcalzan el $100 \%{ }^{23}$.

Tabla 1

Efectividad de la vacuna utilizada en el modelo según la edad de la cohorte del estudio

\begin{tabular}{|l|c|}
\hline \multicolumn{1}{|c|}{ Edad } & Efectividad de la vacuna \\
\hline 1 a 4 años & $100 \%^{16}$ \\
\hline 5 a 9 años & $95 \%^{18}$ \\
\hline 10 a 14 años & $90 \%^{17}$ \\
\hline
\end{tabular}




\section{Epidemiología de la Varicela en Castilla y León ${ }^{15}$}

La tasa de incidencia estimada ha sido de 4.648 casos por 100.000 menores de 15 años (IC: 95\% 4.571-4.726). No se observaron diferencias significativas en la distribución de los casos por sexo. La categorización por grupos de edad mostró que el 93\% de los casos apareció en niños menores de 10 años. El lugar que con mayor frecuencia se produjo la exposición ha sido la guardería/escuela/centro de trabajo. El $12 \%$ de los casos sufrieron algún tipo de complicación, siendo el grupo de 5 a 9 años el más afectado. Las complicaciones más frecuentes fueron manifestaciones cutáneas, conjuntivitis y otitis. Los antihistamínicos sistémicos, seguidos de los antipiréticos fueron los medicamentos más utilizados.

La descripción de la actividad generada durante el proceso mostró que el $32 \%$ de los casos realizaron más de una visita a su médico o pediatra de atención primaria; el $13 \%$ acudieron a los puntos de atención continuada, y el $1 \%$ necesitó al menos una visita domiciliaria. El $4 \%$ de los casos acudió a urgencias hospitalarias en algún momento del proceso y el $0,2 \%$ necesitó hospitalización. La estancia media de las hospitalizaciones relacionadas con la varicela primaria es de 6,5 días ${ }^{8,11,12}$. La media de absentismo escolar para los menores de 15 años fue de 4,1 días. El cuidador principal en este grupo de edad fue el padre o la madre en el $80 \%$ de las ocasiones. La media de días que los padres faltaron al trabajo por la varicela fue de 0,57 días por niño.

\section{Cuantificación de los costes}

Dado que la cohorte se estudia hasta los 15 años, se aplica un factor de descuento a los costes finales de cada persona en función de la edad a la que va a enfermar, basado en la existencia de una preferencia temporal positiva, es decir, la prioridad del presente sobre el futuro. La fórmula para el cálculo del factor de descuento es:

$$
1 /(1+r)^{t}
$$

que muestra el factor de descuento para cada uno de los " $t$ " años del período de estudio y donde " $r$ " es la tasa de descuento utilizada del $3 \%$.

En los estudios de coste-beneficio se calcula el Índice de Beneficio Coste (IBC), magnitud que mide, en términos relativos, el rendimiento de una inversión sanitaria, y que se calcula mediante la relación por cociente entre la suma actualizada de beneficios y la suma actualizada de costes del estudio. Constituye la medida más característica de la rentabilidad (expresada en términos relativos) que es utilizada en el análisis coste/beneficio. Si el IBC es mayor que 1 se interpreta la inversión como rentable; si es igual a 1, como indiferente; y si es menor a 1, como que no resulta rentable. El Índice Beneficio/Coste es un concepto idéntico a la razón o ratio beneficio/coste, que es también conocido como índice de rentabilidad.

\section{RESULTADOS}

El estudio ha sido modelizado mediante un árbol de decisión, con sus ramas y probabilidades (figura 1). En la rama de estrategia de "vacunación", la ausencia de efectividad de la vacuna se ha asumido que la enfermedad se comportará igual que en la estrategia de "no vacunación".

La descripción de todos los costes ocasionados considerados en el estudio en ambas estrategias se exponen en la tabla 2 . Los costes globales y los casos de varicela que ocurren en cada estrategia se exponen en la tabla 3 . 
Figura 1

Árbol de decisión. Estrategias y probabilidades




Tabla 2

Asignación de diferentes costes asociados a la Varicela y a la vacunación

\begin{tabular}{|lr|}
\hline Costes Directos* & \\
Primera Consulta de A.P. o pediatra + tratamiento & 42.59 euros \\
Sucesiva Consulta de A.P. o pediatra & 31.69 euros \\
Punto de Atención Continuada & 44.03 euros \\
Visita Domiciliaria & 52.06 euros \\
Urgencias & 170 euros \\
Hospitalización pediátrica & 340 euros/día \\
Coste estimado vacunación & 34.5 euros/persona \\
Costes indirectos** & \\
Sueldo medio & \\
\hline
\end{tabular}

* Fuente: Direcciones Técnicas de Atención Primaria y de Atención Especializada de la Gerencia Regional de Salud de la Comunidad de Castilla y León, unidades de contabilidad analítica de hospitales de la provincia de Valladolid.

** Fuente: Instituto Nacional de Estadística. Encuesta de estructura salarial 2002. Ganancia media anual por trabajador en Castilla y León.

Tabla 3

Costes totales y casos de varicela

\begin{tabular}{|c|c|c|c|}
\hline \multicolumn{2}{|c|}{ Estrategia no vacunación } & \multicolumn{2}{|c|}{ Estrategia vacunación } \\
\hline & $\begin{array}{c}\text { Coste } \\
\text { (euros) }\end{array}$ & & $\begin{array}{c}\text { Coste } \\
\text { (euros) }\end{array}$ \\
\hline $\begin{array}{l}\mathrm{N}^{\circ} \text { Casos } \\
48.927\end{array}$ & $2.598 .183,04$ & $\begin{array}{l}\mathrm{N}^{\circ} \text { Casos } \\
3.471\end{array}$ & $165.119,01$ \\
\hline $\begin{array}{l}\text { Días trabajo } \\
\text { perdidos } \\
27.888\end{array}$ & $1.908 .522,29$ & $\begin{array}{l}\text { Días trabajo } \\
\text { perdidos } \\
1978\end{array}$ & $83.092,96$ \\
\hline & & Coste Vacunación & 3.450 .000 \\
\hline Coste Total & $4.506 .705,33$ & Coste Total & $3.698 .211,97$ \\
\hline $\begin{array}{l}\text { Beneficios Prog } \\
\text { Vacunación }\end{array}$ & a 258.493 .36 & & \\
\hline IBC* & 1,23 & & \\
\hline
\end{tabular}

* Índice Beneficio Coste.
Según la tasa de incidencia estimada para los niños castellano-leoneses, padecerían la enfermedad casi el $50 \%$ de la población comprendida entre 15 meses y 15 años. La administración de la vacuna de la Varicela junto a la TV, sobre una cohorte de 100.000 niños, evitaría un total de 40.022 visitas al pediatra o médico de Atención Primaria, 6.360 visitas a puntos de atención continuada, 489 desplazamientos de personal médico a los domicilios de los niños enfermos, 1957 visitas a los Servicios de Urgencias Hospitalarios y 98 hospitalizaciones a causa de las complicaciones que la enfermedad puede ocasionar. El número de días de trabajo perdidos evitados a los padres ascendería a 27.888. El programa de vacunación reduciría en un $85 \%$ a un $93 \%$ el número de casos, según el porcentaje de efectividad conseguido por la vacuna en cada grupo de edad.

Los costes médicos directos ocasionados ascienden a 2.598.183,04 $€$ y la pérdida de productividad ocasionada a los padres de los casos de varicela ascendió a 1.908.522,29 €. El IBC de la introducción de la vacuna en el calendario de vacunación infantil se cifra en 1,23.

Análisis de Sensibilidad: Para reducir la incertidumbre que suscitan los estudios

Tabla 4

Análisis de sensibilidad

\begin{tabular}{|l|l|l|l|l|l|}
\hline Factor de descuento & \multicolumn{1}{|c|}{ Efectividad vacuna* $^{*}$} & Días laborables perdidos** & \multicolumn{1}{|c|}{ IBC (social) } & \multicolumn{1}{|c|}{ IBC (costes directos) } \\
\hline \multirow{2}{*}{$3 \%$} & $100 / 95 / 90$ & 0,57 & 1,23 & 0,71 \\
\hline $3 \%$ & $100 / 95 / 90$ & 1,19 & 1,81 & 0,71 \\
\hline $3 \%$ & $100 / 90 / 80$ & 0,57 & 1,19 & 0,66 \\
\hline $3 \%$ & $100 / 90 / 80$ & 1,19 & 1,76 & 0,66 \\
\hline $6 \%$ & $100 / 95 / 90$ & 0,57 & 1,07 & 0,61 \\
\hline $6 \%$ & $100 / 95 / 90$ & 1,19 & 1,57 & 0,61 \\
\hline $6 \%$ & $100 / 90 / 80$ & 0,57 & 1,04 & 0,58 \\
\hline $6 \%$ & $100 / 90 / 80$ & 1,19 & 1,54 & 0,58 \\
\hline $3 \%$ & 100 & 0,57 & 0,65 & 0,38 \\
\hline $3 \%$ & 100 & 1,19 & 0,95 & 0,38 \\
\hline $6 \%$ & 100 & 0,57 & 0,57 & 0,33 \\
\hline $6 \%$ & 100 & 1,19 & 0,83 & 0,33 \\
\hline
\end{tabular}

* \% de efectividad de la vacuna según los rangos de edad: 1-4 años/5-9 años/10-14 años

** Días laborables perdidos por los padres: 0,57 días estimado por la $\mathrm{RMCCyL}^{15}$ y 1,19 días estimado por Díaz-Domingo et al ${ }^{9>}$

*** Dos dosis: se asume una efectividad del $100 \%$ a lo largo de todos los años. 
basados en modelizaciones teóricas, se ha realizado un análisis de sensibilidad para dar mayor robustez y fiabilidad a los resultados y tener una visión más completa de la situación actual. Los resultados obtenidos (tabla 4), modifican los valores de la efectividad de la vacuna, del factor de descuento utilizado y los días de pérdida de trabajo de los padres, y representan su repercusión en la rentabilidad del programa de vacunación. El factor de descuento utilizado en estudios económicos de distintos países es variable, cifrándose fundamentalmente del $3 \%$ al $6 \%$.

\section{DISCUSIÓN}

Desde una perspectiva social, la estrategia de vacunación sistemática infantil frente a la varicela, junto a la TV, se muestra rentable, obteniéndose 23 céntimos de euro de beneficio por cada euro de inversión. Cada caso de varicela en los niños de Castilla y León suponen un coste social de $92,11 €$.

Los resultados de este estudio se sustentan en varias asunciones:

- las tasas de incidencia de la enfermedad se mantienen constantes en la población susceptible y además son iguales en la población vacunada y no vacunada;

- no se han considerado las complicaciones que pudieran producirse como consecuencia de la vacunación, al ser éstas mínimas y de escasa importancia;

- sólo se han analizado las complicaciones registradas por la RMCCyL, no contemplando otras complicaciones e incluso muertes que pudieran ocurrir;

- la efectividad vacunal se ha asumido a partir de las fichas técnicas y estudios realizados en otros países sin tener datos de efectividad en nuestro país o región;
- no se ha tenido en cuenta los posibles beneficios derivados de la disminución de la incidencia de la enfermedad en los sujetos no vacunados, lo que se conoce con el nombre de efecto "rebaño", motivado por una menor circulación del virus.

La repercusión clínica y económica que la enfermedad supone en nuestro entorno se puede evitar a través de programas de vacunación ${ }^{12}$. En los países de clima templado, como España, en los que no se ha introducido la vacunación, se estima que la incidencia anual de la enfermedad coincide con la tasa anual de natalidad ${ }^{10}$. En los Estados Unidos, donde la vacunación frente a la varicela es rutinaria en niños desde 1995, se ha visto un importante descenso en la morbilidad de la varicela 6 años después de la introducción de la vacuna ${ }^{24}$, disminuyendo la circulación del virus salvaje entre la población. También se ha demostrado que la incidencia del Herpes Zoster es menor tras la vacunación que tras la infección natural $^{25}$.

Aunque parece claro el efecto de la vacuna de la varicela a corto plazo en la disminución de la morbilidad y mortalidad, existen importantes dudas respecto a la estrategia de vacunación universal en la infancia. Preocupa fundamentalmente la disminución de la circulación del virus, cuya magnitud dependerá de la cobertura alcanzada, la efectividad de la vacuna y la duración de la inmunidad producida por la misma, y su repercusión sobre la epidemiología del Herpes Zoster, todavía desconocido. Algunos estudios matemáticos predicen que una estrategia de vacunación universal frente a varicela llevará asociado un aumento en la incidencia del Herpes Zoster ${ }^{26,27}$, sin embargo se han publicado recientes estudios que contradicen esta afirmación ${ }^{28}$.

Otro aspecto a tener en cuenta es la disminución de la efectividad de la vacuna con el tiempo. Los estudios realizados hasta la fecha se han desarrollado en un 
contexto en el que el virus salvaje circula ampliamente, lo que puede plantear la más que posible necesidad de incorporar una segunda dosis de recuerdo ${ }^{29}$. Como intento de aproximación a esta situación se ha planteado en el modelo el uso de dos dosis de vacuna y una supuesta efectividad del $100 \%$, siendo el IBC de 0,65. Así mismo se ha visto como varía el IBC modificando los valores de las variables principales, alcanzando 0,95 en la situación más favorable, muy cercano a una rentabilidad positiva, máxime cuando no se han analizado todos los parámetros económicos, como son algunos costes tangibles indirectos, como el transporte. De cualquier modo, si variase la perspectiva del estudio, y sólo se analizasen los costes directos sanitarios, afrontados por el sistema regional de salud de Castilla y León, la aplicación de la vacunación no sería rentable.

Los datos epidemiológicos y de morbilidad usados son similares a otros estudios realizados en España ${ }^{8-12,14}$. El número de días de pérdida de trabajo de los padres utilizado es más bajo que el obtenido en otros estudios, sobre todo europeos ${ }^{14,30}$ y norteamericanos ${ }^{4}$. Este hecho se puede explicar por el actual tipo de núcleo familiar castellano-leonés, donde las mujeres presentan una menor participación del mercado laboral y donde los abuelos ejercen funciones de cuidador en el caso de que trabajen los padres.

En conclusión, la varicela se asocia a complicaciones graves que comportan un importante coste terapéutico y de hospitalización. Existe una vacuna eficaz para prevenir la enfermedad, y la vacunación universal reduciría el número de casos, su gravedad, los gastos directos generados y los gastos indirectos sociales. No obstante, las decisiones sobre la aplicación y extensión de los programas de salud deben basarse no sólo en criterios de efectividad, sino también de eficiencia. En los últimos años ha existido un interés cre- ciente en determinar la elevada morbilidad de la varicela y sus complicaciones, así como el elevado coste social que produce en las distintas Comunidades Autónomas de España. A pesar de las recomendaciones de la instauración universal de la vacuna, se dispone de muy pocos estudios que analicen la rentabilidad de dicha medida. Este trabajo aporta una información útil para la toma de decisiones a la hora de la distribución de los recursos sanitarios entre los diferentes programas preventivos.

\section{AGRADECIMIENTOS}

A las Direcciones Técnicas de Atención Primaria y Especializada de la Gerencia Regional de Salud de Castilla y León y a la Red de Médicos Centinelas de Castilla y León.

\section{BIBLIOGRAFÍA}

1. Forcén T, Garuz R, Cabasés J, Ruiz de Ocenda M, Martínez JA, Izko J. Evaluación económica de la vacunación rutinaria a niños de 15 meses de edad frente al virus de la varicela-zoster. An Sist Sanit Navar. 2000;23(1):115-124.

2. Thiry N, Beutels P Tancredi F, Romano L, Zanetti A, Bonanni $\mathrm{P}$ et al. An economic evaluation of varicella vaccination in italian adolescents. Vaccine. $2004 ; 22: 3546-3562$.

3. Lin F, Hadler JL. Epidemiology of primary varice1la and herpes zoster hospitalizations : the pre-vaccination era. J Infect Dis. 2000;181 :1897-1905.

4. Lieu TA, Cochi SL, Black SB, Halloran ME, Shiefiled HR, Holmes SJ et al. Cot-effectiviness of a routine varicella vaccination program for US children. JAMA. 1994;271:375-381.

5. An Advisory Committee Statement (ACS). National Advisory Committee on Immunization (NACI) Update on Varicella. CCDR. 2004. ACS-1.

6. Vázquez M, LaRussa PS, Geershon AA, Steimberg SP, Freudigman K, Shapiro ED. The effectiviness of the varicella vaccine in clinical practice. N Engl J Med. 2001;344:955-960. 
7. Wise RP, Salive ME, Braun MM, Mootrey GT, Seward JF, Rider LG et al. Postlicensure safety surveillance for varicella vacine. JAMA. 2000;284(10):1271-1279.

8. Gil A, González A, Oyagüez I, San Martín M, Carrasco P. Burden of hospitalizations attributable to rotavirus infection in children in Spain, period 1999-2000. Eur J Epid. 2004; 19: 699-702.

9. Díez-Domingo J, Aristegui J, Calbo F, GonzálezHachero J, Moraga F, Pena J, et al Epidemiology and economic impact of varicella in immunocompetent children in Spain. A nation-wide study. Vaccine. 2003; 21:3236-3239.

10. Guillén JM, Hernández-Barrera V, Pérez M, González A, Gil A. Hospitalizaciones atribuibles a infecciones por virus varicela-zoster en la Comunidad Autónoma de Madrid, en el período 20012003. Vacunas. 2006;1: 17-22.

11. Pérez-Yarza EG, Arranz L, Alustiza J, Azkunaga B, Uriz J, Sarasua A et al. Hospitalizaciones por complicaciones de la varicela en niños menores de 15 años. An Pediatr (Barc). 2003;59:229-233.

12. Piqueras AI, Otero MC, Pérez-Tamarit D, Asensi f, Diosdado N, Santos M. Hospitalizaciones por varicela en el hospital infantil $\mathrm{La} \mathrm{Fe}$ (Valencia 2001-2004). An Pediatr (Barc). 2005;63:120-124

13. Ministerio de Sanidad y Consumo. Dirección General de Salud Pública. VARICELA: Epidemiología y situación actual. Vacunas: características y eficacia/efectividad. Recomendaciones de vacunación y sus implicaciones en Salud Pública. 2005

14. Díez-Domingo J, Ridao M, Latour J, Ballester A, Morant A. A cost benefit analysis of routine varicella vaccination in Spain. Vaccine 1999;17:13061311.

15. Gil Costa M. Varicela. Inf Red Med Centinelas Castilla León 2005;59:5-8.

16. Weibel Re, Neff BJ, Kuter BJ, Guess HA, Rothenberger CA, Fitzgerald AJ, et al. Live attenuated varicella virus vaccine. Efficacy trial in healthy children. N Engl J Med 1984; 310: 1409-15.

17. Agencia Española del Medicamento(AEM). Fichas técnicas. Resumen de las características del producto VARIVAX. Available to: http://sinaem.agemed.es: 83/presentacion/principal.asp. Revisado 08/02/2006.

18. Kuter BJ, Weibel RE, Guess HA, Mattheus H, Morton DH, Neff BJ et al. Oka/Merck varicella vaccine in healthy children: final report of a 2 - year efficacy study and 7 year follow up studies. Vaccine 1991;9:643-647.

19. Gail K, Lee B, Strine T, Carraher C, Baughman $\mathrm{AL}$, Eaton $\mathrm{M}$ et al. Outbreak of varicella ata daycare center despite vaccination. N Engl J Med 2002;347:1909-1915.

20. Gail K, Fair E, Mountcastle N, Britz P, Seward J. Young age at vaccination may increase risk of varicella vaccine failure. J Infect Dis. 2002;186:102-105.

21. Asano Y, Nagai T, Miyata T, Yazaki T, Ito S, Yamanishi $\mathrm{K}$ et al. Long term protective immunity of recipients of the OKA strain of live varicella vaccine. Pediatrics. 1985;75(4):667-671.

22. Johnson CE, Stancin T, Fattlar D, Rome LP, Kumar ML. A long term prospective study of varicella vaccine in healthy children. Pediatrics. 1997; 100(5):761-766.

23. Díez MT, Rodríguez MJ. Coberturas de vacunación sistemática infantil en Castilla y León año 2003. Bol Epidemiol Castilla León. 2004; 20.

24. Seward JF, Watson BW, Peterson CL, Mascola L, Pelosi JW, Zhang JX et al. Varicella disease after introduction of varicella vaccine in the United States. 1995-2000. JAMA. 2002; 287(5): 606-611.

25. Skull SA, Wang EE. Varicella vaccination-a critical review of the evidence. Arch Dis Child. 2001; 85(2):83-90

26. Shuette MC, Hethcote HW. Modeling the effects of varicella vaccination programs on the incidence of chickenpox and shingles. Bull Mathematical Biol. 1999; 61:513-564.

27. Brisson M, Edmunds WJ, Gay NJ, Law B, De Serrres G. Modelling the impact of immunization on the epidemiology of varicella zoster virus. Epidemiol Infect. 2000; 125: 651-669.

28. Jumaan AO, Yu O, Jackson LA, Bohlke K, Galil $\mathrm{K}$, Seward JF. Incidence of herpes zoster, before and after varicella-vaccination-associated decreases in the incidence of varicella, 1992-2002. J Infect Dis. 2005; 191: 2002-2007.

29. Vázquez M, LaRussa PS, Gershon AA, Niccolai LM, Muehlenbein E, Steinberg SP et al. Efectiveness over time of varicella vaccine. JAMA. 2004;291:851-855.

30. Beutels P, Clara R, Tormans G, Van Doorslaer E, Van Damme P. Cost and Benefits of routine varicella vacunation in German Children. J Infec Dis. 1996;174 Suppl 3:S335-341 
